\title{
Erratum to: What to do in globalised economies if global governance is missing? The vicarious role of competition in social responsibility
}

\section{Leonardo Becchetti • Giorgio Federico • Nazaria Solferino}

\section{Erratum to: Int Rev Econ (2011) 58:185-211 \\ DOI 10.1007/s12232-011-0122-0}

Unfortunately, the author names are listed incorrectly in the original publication of the article. The correct version of the author group is mentioned below:

Leonardo Becchetti · Giorgio Federico · Nazaria Solferino

The online version of the original article can be found under doi:10.1007/s12232-011-0122-0.

L. Becchetti $(\bowtie)$

Dipartimento di Economia e Istituzioni, Facoltà di Economia, University of Rome Tor Vergata,

Via Columbia 2, 00133 Rome, Italy

e-mail: becchetti@economia.uniroma2.it

G. Federico

Credit Agricole SA, FIN/TX, Evergreen AQUA/2/612B, Paris Cedex, France

N. Solferino

Università della Calabria, Campus di Arcavacata, via Pietro Bucci,

87036 Arcavacata di Rende (CS), Italy 